\section{Clinital a decture}

ON THE

\section{TREATMENT OF CARBUNCLE.}

\author{
By JAMES PAGET, D.C.L., F.R.S., \\ SURGEON TO ST. BARTHOLOME'S HOSPITAL. \\ (Shorthand Report, revised by the Author.)
}

GENTLEMEN,- - You have recently had the opportunity of seeing four cases of carbuncle treated in my wards after methods which you would probably describe, if asked to do so, by saying that "nothing was done for them." Here are the patients' papers; and there is, to be sure, no medicine set down for them to take, and you know that no surgery was inflicted on them,---and yet a good deal was done for them, though the treatment was what does commonly pass by the name of "doing nothing." They were carefully fed, washed, cleaned, and bedded; and their carbuncles were very skilfully dressed and washed with proper things, and every care was taken to shut out all untoward influences from them. And if any complication in their cases had arisen, these would have been immediately met. But no complications occurred; and, therefore, the cases passed through their course without treatment, as it is said-that is, without medicine, and with no surgery, no active surgery, no incisions or anything of that kind. And since all these cases passed through their course very favourably, and all the patients were, or will be, discharged at a comparatively early time after their admission into the hospital, I will take this occasion of giving you some observations on the manner of treating this disease.

Although you may not have seen much of it, you must all have heard of the ordinary manner in which carbuncles were treated formerly, and still are by some; a method which consists mainly in making large incisions through them, and giving very large quantities of food and stimalants, as well as considerable doses of quinine, bark, and other tonics. I do not at all mean to say that the things which in these cases I left undone would have done any harm; but what I hold of them is, that they would have been quite useless, and some would have been sources of great discomfort to the patients. And in the way in which I speak of these things you may notice that I exemplify that rule which I have always impressed upon you, of asking yourselves, when you seem to have been successful with some medicine, "What would have happened if I had not given it ?" The apparent consequence of giving a medicine may be plain enough; but you cannot too of ten repeat to yourselves the question-as a rule, I will not say of practice, but of the study of your own practice,- "What would have happened if this or that, which seems to have been successful, had not been done?"

First, with regard to the incisions made in carbuncles. The ordinary plan, still recommended by some, is, as soon as a carbuncle is seen, to make two incisions crucially from border to border. It is said that they must go even beyond the edges of the carbuncle into the adjacent healthy textures. I have not followed that method of practice very often, but I have followed it quite often enough to be sure that it does not produce the effects which are commonly assigned to it. It is commonly said that if you will thus make crucial incisions into a carbuncle, you will prevent it spreading. If you can find a carbuncle two or three days old, and cut that right across in both directions, I think it very likely that you will prevent it spreading. But even therein is a fallacy; for there is no sign by which, on looking at a commencing carbuncle, you can tell whether it will spread or not, whether it will have a diameter of an inch, or of three, six, or ten inches. The question, therefore, that I spoke of comes back, "What would have happened if I had not made these incisions?" And the answer to that question will be rather according to temper than according to knowledge. For as I watch men in their conclusions upon such cases as that, I No. 2368 . generally find that self-satisfaction says, "I saved the man's life;" self-dissatisfaction, "I did him no good." The truer scientific temper stands midway, and says, "I will wait for further information on the matter-till I have seen more cases, and then decide whether, in the earliest stages of carbuncle, incisions are useful or not." After this time of three or four days $I$ have seen sufficient numbers of carbuncles thus divided, and have divided enough for myself, to say that it will not hinder the spreading. I have seen carbuncles spread in as large a proportion of cases after incisions as in cases that have never been incised at all. I have in $m y$ mind a striking case that occurred to me early in practice, when I followed the routine, and, in a friend of my own, divided a carbuncle most freely. I cut it after the most approved fashion in depth and length and width, and then it spread. After two or three days more all the newlyformed part was cut as freely as the first, and then it spread again, and again it was cut as freely. Then it spread again, and was not cut. Then, in a natural time, it ceased to spread, and all went on well. These are only general impressions that I give you, because one cannot count the cases in which cutting has been practised, and those similar cases in which it has not; nor even then could it be said whether those in which the cutting was practised would have spread if left alone. On a very strong general impression, however, I say that carbuncles will spread after cutting in as large a proportion of cases as they will spread in without cutting.

Then it is said that carbuncles are relieved of their pain if they are thus very freely cut. Here, again, however, is only a partial truth. A carbuncle of two or three days' standing, which is hard, tense, and brawny, is very painful; and cutting it will relieve, in many cases, a considerable portion of the pain. But after this, when the carbuncle begins to soften, and when pustules begin to form upon its surface, and pus in its interior, it ceases to be painful of its own accord, and without incisions. Thus there are two distinct stages of carbuncle in reference to the pain; the early stage, when it is hard and still spreading, and is generally intensely painful, and the later stage, in which that pain nearly ceases. A carbuncle divided in the first stage, in the first two or three days of its existence, may be relieved of some of its pain; if divided in the later stage, what little pain may exist is altogether unaffected by the cutting. And even cut as you may, you cannot always put aside the extreme pain that a carbuncle sometimes has, even to its later time. Some two or three years ago, I was called to a member of our profession with a large carbuncle in the middle of his back. His friends had been much alarmed about the state of his mind, for he had been suffering great mental anxiety for some time, and they were in fear lest the excessive pain of the carbuncle should, in its disturbed state, do his mind permanent damage. So they persuaded me to cut it, and I cut it after the old plan, very wide across, and far into the adjacent textures, as freely as could be. It did not in the least relieve him. I never saw a carbuncle through its whole course so painful as that was, and up to the last, till the healing was nearly completed, he suffered more or less pain in it. So that the conclusion in reference to pain must be this: if they can be divided in the first three or four days, while still hard and brawny, it may relieve some measure of the suffering; at a later period the incisions have no influence at all.

The third point is stated thus, that by the incision of carbuncles you accelerate their healing, giving facility for the exit of sloughs. But herein is the greatest fallacy of all. When the cutting of carbuncles was more customary in this hospital than it is now, when I did not cut them, and some of my colleagues did, I used to be able to compare the progress of cases cut and of cases uncut, and time after time it was evident that the cases uncut healed more readily than those cut. A man who is now in the hospital I have brought round here that I may illustrate this point to you. This is the man, Timothy C-, aged fifty-five. When he came in, his carbuncle had a length of more than six inches, and a breadth of three and a half; and it formed the ordinary hard, compact, tense, and brawny mass that a carbuncle usually does. It had at that time already taken to suppurate, and little pustules were pointing on the surface. If I had followed the practice of incisions, I should have had to make a cut in one direction of about seven inches, and in the other of about five, and after that I should have had not only the wounds wide, open, and gaping, and having 
themselves to heal, but a great part of the substance of the carbuncle fairly exposed, and also under the necessity of healing. But you will observe that the whole of the space that now remains to heal is a series of openings in the middle of the carbuncle, through which the sloughs are to be separated, through which, indeed, nearly the whole of the sloughs have already been discharged, and which now merely remain to be healed like the cavities of small abscesses. In that way you narrow greatly the extent of wounded surface to be healed. Indeed, it by no means always follows that the whole carbuncle, or its whole base, sloughs. Carbuncles, if not divided, not unfrequently only suppurate about their centres, and slough only in their central parts, and the borders merely clear up by the softening and dispersion of the inflammatory products in them. In every case of that kind you save greatly the amount of healing which has to be gone through. Nay, in some cases carbuncles completely abort. One of these cases, of which I have the paper on the table, was that of a woman, aged sixty-four, who came in with a carbuncle nearly as large as this, in a condition which, it might be said, required incision at once; but, with the exception of two or three small points, no suppuration or sloughing ensued. That carbuncle dispersed, aborted, cleared away. This case shows the more ordinary course of events-the sloughing of the central part, the gradual discharge of the sloughs, and the comparatively small spaces which are left in the centre of the carbuncle as the sole space in which the process of healing has to be achieved.

On these three points, which are the grounds that have been assigned as reasons for cutting carbuncles, I have now given you the evidence on which I have ceased from the practice. I fully believe that crucial incisions do not prevent extension; that it is only a limited set of cases in which the incisions diminish pain; and that with regard to the time that is occupied in healing with or without incisions, the healing without incisions is very clearly and certainly a great deal the quicker.

The kind of incisions that I have been speaking of is the old plan of crucial incisions. Another method which I have occasionally tried, but of which I can only state the same general results, is that of subcutaneous incision. This has been supposed to have the same general effect as the other; and I think that the same general conclusions may be drawn respecting it: that it is a measure unnecessary in the treatment of carbuncle, and that it retards rather than hastens the healing. When I speak thus of the incision of carbuncles, however, I do not mean to say that there is no condition of carbuncle in which an incision is not useful. Sometimes a carbuncle sloughs in its central part, with one continuous slough of integument holding in a quantity of pus. In that case you would cut through the slough, or through any adjacent part of the carbuncle, to let out the pus, as you would open an ordinary abscess. But this is not a measure which is commonly understood by the "incision of a carbuncle."

If you ask why one may not cut a carbuncle though it may do no good, I reply that you should never be actively useless, and that there are some cases in which the cutting does considerable harm. Carbuncles, for the most part, occur in persons broken down in health, exhausted by overwork, or by bad food, or in general deteriorated health-as sometimes in diabetos or albuminuria; and in all these states it is a good general rule to save the blood they need for healing. The loss of blood from the carbuncle itself would not be considerable; the hard substance of the carbuncle, when cut into, does not bleed, or bleeds but little. But to carry out the incision perfectly, you have to cut into the adjacent healthy texture; and this sometimes bleeds very profusely, so as to lead to all the distress and pain of plugging the wound with this or that substance to arrest the blood.

Another measure in the treatment of carbuncles which is supposed to be necessary, is very high feeding and large quantities of stimulints. I learned the opposite of this in one of those cases which you will do always well to studythose, namely, in which the patient refuses to do what you advise him. It is from such cases that we may often learn what is commonly called the "natural history of disease" its course undisturbed by treatment. A case occurred to me once of an old gentleman, eighty years of age, who had a car'suncle, as big as it could be, on the back of his neck, for it extended from one ear to the other, and from his occipital spine to the third cervical vertebra. He measured it for his own amusement, and it was fourteen inches over its surface transversely, and nine inches vertically-a carbuncle, then, of the largest size, and one, it might have been supposed, attended with considerable risk to life. I urged him very strongly to take a large quantity of what is called "support," for I was at that time under an impression of its necessity. He absolutely refused, however, and nothing would induce him to take it. I was therefore content to stand by and study the natural history of disease in this huge earbuncle; and the natural history of it was a history that one would have wished to witness in every carbuncle of its size, for no case could pass through its course in a better method. He led his ordinary abstemious life, took moderate quantities of food and of stimulant, lived through a carbuncle of the greatest severity, and finally made a complete recovery, and lived for several years after.

Another case which impressed me very much was that of a friend of my own in the profession, who had a carbuncle on the back of his neck, of very considerable size. Sir Benjamin Brodie and Mr. Stanley attended him with me, and under their advice the carbuncle was cut. I watched its course afterwards, and felt sure that the cutting had done neither good nor harm. It went on as carbuncles do when not cut. But the gentleman was subject to intense headaches, of which he knew by experience the only possible remedy was almost entirely to leave off food, and absolutely and entirely to leave off stimulants. One of these headaches occurred during the course of the carbuncle, at a time when we had put him upon very full diet and abundant stimulant. He said then that he must leave off his stimulants and food, and we looked with some alarm at what would be the result on the progress of the carbuncle. I remember $\mathrm{Mr}$. Stanley saying to him, in his distinct manner, "My dear fellow, if you don't take food, you will die." "Very well," he said, " then I will die, but I will not take food and increase my headache." According to his own wish, therefore, we reduced his diet to a very low level. The course of the carbuncle was not affected at all, unless it were for good; and after three or four days of this, which might be called comparative starvation, he described himself in his own emphatic fashion as being " as jolly as a sand-boy."

Since that time I have watched carefully all cases that I have seen, and I am certain that there is no good to be obtained by large feeding or abundant stimulants in ordinary cases of carbuncle. The whole of these cases that have been in the hospital were put on our ordinary meat diet, with a pint of porter daily; and I see that two of them have had four ounces of wine a day, one of these being a person aged sixty-four and the other sixty-three, and both having carbuncles of considerable size. You will find that for patients in private life it will do very well if you tell them that they may have about two-thirds of their ordinary amount of food, and about the same proportion of their ordinary quantity of stimulants. But indeed there is scarcely any reason to change in any material degree the ordinary mode of life of a patient with carbuncle. So far as he can with comfort take that to which he is accustomed, so far he may. If his diet has been habitually low, so it may remain; if habitually high, so, within certain limits and somewhat reduced, it may still remain.

Now you may ask what I should set down as the things to be done for a carbuncle. These boards, nearly bare as they are, may tell you. In local treatment one of the best things you can do, if the carbuncle is small, is to cover it with emplastrum plumbi spread upon leather, with a hole in the middle through which the pus can exude and the slough can come away. That, occasionally changed, is all the covering that a small carbuncle will need. It is difficult thus to cover the whole surface of a large carbuncle, and to keep it clean; therefore, I think that the best application for that is the common resin cerate. This should be spread large enough to cover the whole carbuncle, and over it should be laid a poultice of half linseed-meal and half bread. And, if you want to exercise your skill, learn to make that poultice well, and to put it on well, and to keep it in its place well. That mode of dressing the carbuncle, so far as the materials are concerned, will last through its whole course; but whilst the carbuncle is making progress and discharging its slough, you will find plenty of room for 
the exercise of considerable skill in dressing it, and filling up the cavities with soft substance spread with this ointment. Besides this, the carbuncles are to be carefully washed, especially with some deodorising substance, as Condy's fluid, or weak carbolic acid, and the cavities may be syringed out with it. The importance of cleanliness is very great. You noticed in the man whom I showed you just now the spots of acne and boils around the edges of the carbuncle. This points out the necessity of care, which I suppose had not been taken there, to keep the surface of the skin adjacent to the carbuncle perfectly dry, and free from any contact with the discharge, which seems really to have the power of infecting the neighbouring skin, and so producing the boils which are apt to arise, sometimes in clusters, around the carbuncle. Of diet $I$ have already spoken to you. Of medicines I say nothing. Quinine, bark, and other medicines of that class, may be given if you please, or in case of evident need, and so may aperients; but there is really no need of them in an ordinary case of carbuncle. But there is one medicine which you may find very valuable, and that is opium, especially in all the earlier painful stages of carbuncle, in which it relieves the suffering as thoroughly as incisions, or anything I know. After the early stages, even that is unnecessary, except for some patient who may be unable to sleep.

But there is one measure in the treatment of carbuncle which is seldom employed, and yet is of great importance, and that is letting the patient have very free air. The general idea that carbuncles are very dangerous diseases has commonly led to the patients being entirely confined to bed and kept shut up in their rooms. There is in that an unnecessary care; and this, too, I learned from a patient who refused to comply with injunctions-a gentleman with a large carbuncle on the back of his head, who would not keep his bedroom. He had been accustomed to an active life, and after seventy or eighty years of that custom he was quite indisposed to remain in his room. So with that carbuncle he daily came down stairs, changing his room and moving about the house as well as the pain and weakness would allow him. No carbuncle could go on better; all the stages were passed through without any risk or trouble, and it healed with unusual speed. After that I had a yet more striking case. A lady came to London "for the season," as she called it; and she had not been here more than a week or ten days before a carbuncle came out on the back of her head, just under her hair. It was a great vexation to her that she had to give up all her amusements; and so, as she did not mind the pain, she the last, I saw any value in a "chignon." She dressed her carbuncle under the chignon, and she went to the park, to the theatre, and to dances unharmed, and with her carbuncle quite unseen, and no trouble whatever followed. It healed up after the ordinary fashion in about the ordinary time. But, indeed, you may see cases of this description on a much larger scale if you watch the carbuncles that come to us in the out-patients' room. There we often see them of considerable size, and they do as well among the out-patients as among the in-patients; and yet these outpatients are freely in the air all day, and many of them continue at their work. So you may set it down as one point to be attended to in the management of carbuncles that patients should not be confined to their room. They should at least have change of air in their own house; and, unless they are too low, they should not avoid exposure to the fresh open air.

Treating your cases of carbuncle upon this plan, I believe you will find that the great majority will pass through their course well. I cannot tell you what the ordinary proportion of deaths from carbuncle is; but I know that carbuncles are commonly looked upon in the profession as dangerous things, and a large carbuncle on the back of the head is considered to be fraught with risk to the patient's life. But that is very far from being the case in my experience. Remembering, as far as I can, or rather guessing at the number of carbuncles I have had to treat, I should say that there is no other disease of the same extent and general severity which is attended with so little risk to life. During twenty years of hospital and private practice, I cannot have treated less than 200 carbuncles; and of these 200 , four have died, giving a mortality, at a fair guess, of only two per cent.-a mortality which is less than that of most of the minor ope- rations of surgery, and less really than that of any disease of equal severity that you can name. Of those four deaths, one occurred in a patient aged seventy-eight, who died of erysipelas after the carbuncle had nearly healed. Another was a gentleman of about fifty-five years of age, who died of chronic pyæmia. The third was a gentleman aged fifty, who died with acute pyæmia. And the fourth was a patient of about fifty years of age, who died rapidly exhausted. The first three were from causes which may almost be called accidental; for so we call them when occurring after an operation, and it would be unreasonable to suppose that any other method of treatment would have averted the consequences. The other died, possibly, on account of the deficient stimulation; for he was a man who had lived freely, and took during treatment less than he had been accustomed to have. The main point, however, to which I wish to direct your attention, is that the mortality may be as little as two per cent. I cannot doubt that the mortality was considerably larger when carbuncles were severely cut; for the severe cutting meant often severe bleeding, and was attended with all the consequences of large wounds. Thus, though I do not know the exact proportion, I believe that the general reputation of the danger of carbuncle was well founded, and that among the reasons for the diminished mortality of carbuncles may be set down as chief, the more frequent avoidance of the custom of cutting them.

Speaking of the mortality of carbuncle, however, I must remind you that I am not speaking of a disease which sometimes passes under the name of carbuncle-the carbuncular inflammation of the lip which sometimes occurs in young persons: a disease which you may not have seen, and may pass many years without seeing. It was described by a former house-surgeon of this hospital, Mr. Harvey Ludlow, as malignant pustule of the lip. Dr. Budd, of Bristol, has also so described it. Commencing at one spot, inflammation of the whole lip follows and spreads to the face, and then disease of the lymphatics ensues, with pyæmia as its consequence. It is a disease so unlike carbuncle that it ought not to be known under the same name. It seems to me not like the accounts given of the malignant pustule abroad, and $I$ have seen no other disease like it in England. It attacks especially young persons from fifteen to twenty-one, and of fifteen cases that I have seen, only one recovered. That disease is not carbuncle, nor is the mortality of that disease to be counted in estimating the mortality from carbuncle. Ordinary carbuncle on the lip and face has none of those special characters, and is not more fatal in those situations than in any other.

\section{Gbstract of a difecture ON THE}

\section{USE OF BRUCINE IN STOMACHAL EPILEPSY.}

By J. SPFNCE RAMSKILL, M.D. LoND., PHISICIAN TO THE HOSPITAL FOR PARAIYSIS AND EPILEPSY; PHYSICIAN TO, AND LECTURBR ON MEDICINE AT, THE LONDON HOSPITAL.

A LARGE proportion of the cases of epilepsy have, as an aura, or warning of the coming fit, some disturbance referred to the stomach-such as nausea, attacks of flatulence, undefinable sensations, represented as rising upwards, and consciousness ceasing when the sensation reaches the head; and these are the cases, also, in which vomiting follows the fit. The vomited matters always contain food, and sometimes in an undigested condition, although some hours may have elapsed since the last meal. There may be no proper symptoms of dyspepsia in the intervals between the fits beyond a constant disposition to the generation of flatus, indicating permanent disorder of the ganglionic nerves. The functions of the pneumogastric, moreover, become perverted; and the cause of this may be at the root of the nerve, or at its periphery. Supposing disorder of the centre 